\title{
Gravitational lensing by the supermassive black hole in M31
}

\author{
V. Bozza*, S. Calchi Novati, L. Mancini \\ Dipartimento di Fisica "E.R. Caianiello", Università di Salerno, via S. Allende, Baronissi (SA), \\ Italy. \\ Istituto Nazionale di Fisica Nucleare, Sezione di Napoli, Italy. \\ E-mail: valbozesa.infn.it, novatiesa.infn.it, lmanciniesa.infn.it
}

\begin{abstract}
We examine the possibility of observing gravitational lensing effects caused by the supermassive black hole in the center of M31. The sources can be provided by stars belonging to the bulge and the disk of M31, but also by the central clusters recently identified around the supermassive black hole. For each stellar population we model the spatial distribution and the luminosity function in great detail. This allows us to derive precise estimates for the number of sources whose secondary image is more luminous than a given threshold, fixed by observational conditions. We present estimates in the $K$ and $V$-bands along with several distributions characterizing gravitational lensing, such as the angular separation and velocity of the secondary images with respect to the black hole, and the distribution in the source luminosity and distance. We finally suggest a strategy for the identification of lensing candidates, specifying the instrumental requirements for the observations, and discuss the physical relevance of the new independent information obtained by gravitational lensing detections.
\end{abstract}

The Manchester Microlensing Conference: The 12th International Conference and ANGLES Microlensing Workshop

January 21-25 2008

Manchester, UK

\footnotetext{
* Speaker.
} 


\section{Introduction: Gravitational lensing by supermassive black holes}

It is widely accepted that many galaxies host supermassive black holes (SMBH) in their centers. Estimated masses range from $10^{6}$ to $10^{10}$ solar masses [1]. It can be naturally guessed that gravitational lensing effects due to such massive bodies should be large enough to be observed, at least for those black holes that are closer to us.

The closest SMBH is right in the center of our own Galaxy. It has been recognized in the radio source Sgr A* and its mass has been estimated to $3.6 \times 10^{6} \mathrm{M}_{\odot}$ by dynamical measurements of stars orbiting it [2]. The current value for the distance between the sun and the center of the Galaxy is $8 \mathrm{kpc}$. The first authors who studied gravitational lensing by Sgr A* were Wardle and YusufZadeh [3] who guessed that several bulge stars should be lensed at the same time. More precise estimates have been presented by several authors $[4,5,6]$, who argue that about 10 bulge stars should have a secondary image brighter than $K=23$. Alexander undertook a search for possible candidate pairs with the aim of using gravitational lensing for pinpointing the gravitational center of the galaxy [7]. A more subtle lensing effect is the astrometric shift of background stars due to light bending by $\mathrm{Sgr} \mathrm{A}^{*}[8]$.

A very interesting chance of observing gravitational lensing by Sgr A* is offered by stars orbiting very close to it. For several of them we have a considerably precise knowledge of the orbital parameters [2]. This encourages the search for possible alignments of these stars behind or in front of the central black hole, which would render the secondary image bright enough to be detectable. Several studies have provided precise predictions for the peak luminosity and position of the secondary images of such stars $[9,10,11]$. Gravitational lensing of these stars assumes a particular interest because the secondary image would be visible even in a regime in which the light deflection is not small and next-to-leading order terms in the deflection angle come into play [12].

With respect to $\mathrm{Sgr} \mathrm{A}^{*}$, gravitational lensing by other SMBH is obviously disadvantaged by the fact that their distance is much larger. Nevertheless, as will be clarified in the next section, the black hole in M31 and its environment present some interesting peculiarities that motivate a thorough study of gravitational lensing probability. In Section 2 we describe the core of M31 and our efforts to model it as precisely as possible. In Section 3 we present our estimates for the expected number of secondary images above a given threshold. In Section 4 we give some details about the phenomenology of gravitational lensing by showing some interesting distributions. In Section 5 we discuss observational perspectives and draw our conclusions. We refer to Ref. [13] for a more detailed report of our work.

\section{The core of M31}

M31 is the closest galaxy to the Milky Way (excluding small satellites). Its distance is 760 $\mathrm{kpc}$, that is nearly 100 times larger than the distance to $\operatorname{Sgr} \mathrm{A}^{*}$. On the other hand, the central black hole of M31 is believed to have a mass of $1.4 \times 10^{7} \mathrm{M}_{\odot}$, i.e. four times larger than Sgr A*. Possible sources for gravitational lensing are mainly provided by bulge and disk stars. In addition, the core of M31 is characterized by an interesting non-trivial structure. Two clusters of stars, named P1 and P2, lie in the middle of M31 [14]. These two apparently distinct clusters can be interpreted as the apocenter and the pericenter of the same eccentric disk [15]. Moreover, a third cluster of young 
blue stars has been detected within P2 and has been named P3 [16]. Stars in P3 are very close to the central black hole and have very high rotation velocities. The existence of these clusters of stars orbiting very close to the SMBH in M31 motivates a deep investigation of the gravitational lensing probability, which can be expected to be enhanced by such a concentration of sources around the lens.

In our study, we have calculated the gravitational lensing probability of four stellar populations: the disk of $\mathrm{M} 31$, the bulge, the eccentric cluster $\mathrm{P} 1+\mathrm{P} 2$ and the inner cluster P3. In order to obtain the most accurate estimates, we have performed a very careful modelling of these four populations using the available data for the spatial distributions and the luminosity functions. In particular:

- For P3 we have followed Bender et al. [16], who argue that P3 is composed by young blue stars born in a single star formation episode occurred $200 \mathrm{Myr}$ ago. The luminosity function has been synthesized according to this hypothesis using the program IAC-STAR by Aparicio $\&$ Gallart [17]. For the spatial distribution we assume a thick disk model with radius $0.8 \mathrm{pc}$ and height scale $0.1 \mathrm{pc}$.

- For P1+P2 we have followed Peiris \& Tremaine [18], who reproduce the observed isophotes by generating $10^{7}$ stars in random orbits with average semiaxis $1.37 \mathrm{pc}$ and eccentricity 0.5 . We have assumed that the luminosity function is the same as that for the bulge.

- The bulge spatial distribution can be deduced by the isophotes, as reported by Kent [19]. The bulge scale is about $1 \mathrm{kpc}$. The luminosity function has been synthesized by the program IAC-STAR using the metallicity distribution of Ref. [20].

- For the disk spatial distribution we have followed Ref. [21], adopting a radius of $5.5 \mathrm{kpc}$ and a height scale of $0.3 \mathrm{kpc}$. The star formation rate and the metallicity of the disk are assumed to be constant [22] in the generation of the luminosity function.

Each source population is thus characterized by a spatial distribution $f_{P_{j}}(x, y, z)$ normalized to unity (with $P_{j} \in\{\mathrm{P} 3, \mathrm{P} 1+\mathrm{P} 2$, Bulge, Disk $\}$ ) and a luminosity function $n_{P_{j}}\left(M_{B_{i}}\right)$ for each spectral band $B_{i}$. We have focused on the $K$ and $V$-bands. The $K$-band is the band for which present infrared interferometers are optimized. Therefore it is the most promising one for the detection of secondary images forming very close to the SMBH. The $V$-band provides a useful comparison. For reasons of space, here we just report the results in the $K$-band, referring to Ref. [13] for those in the $V$-band. The luminosity functions are normalized in such a way that $n_{P_{j}}\left(M_{B_{i}}\right) d M_{B_{i}}$ is the number of stars with absolute magnitude in the range $\left[M_{B_{i}}, M_{B_{i}}+d M_{B_{i}}\right]$.

\section{Number of lensed sources}

Basic gravitational lensing formulae can be used to calculate the luminosity of the secondary image generated through gravitational lensing by the central black hole of M31 for a given source at distance $D_{\mathrm{LS}}$ from the black hole and absolute magnitude $M_{K}$. We indicate by $K_{\mathrm{thr}}$ the maximum magnitude a secondary image can have in order to be detected by a given observational setup. Then, for any given source magnitude $M_{K}$, it is easy to define the region of space where the source must 
(a)

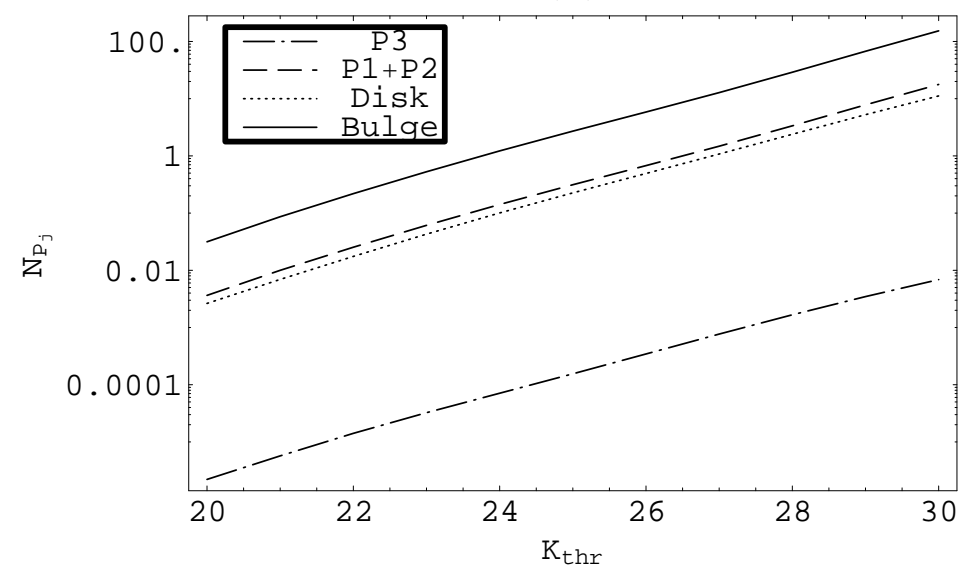

Figure 1: Number of expected lensing events as a function of the threshold magnitude for the secondary image in the $K$-band.

lie in order to have a secondary image above the threshold $K_{\mathrm{thr}}$. The boundary of such a region (that we call lensing zone) can be calculated by basic gravitational lensing formulae; see [13] for further details.

The probability that a source with magnitude $M_{K}$ belonging to the population $P_{j}$ has a secondary image more luminous than $K_{\mathrm{thr}}$ is thus

$$
\Pi_{P_{j}}\left(M_{K}, K_{\mathrm{thr}}\right)=\int_{L Z\left(M_{K}, K_{\mathrm{thr}}\right)} d x d y d z f_{P_{j}}(x, y, z),
$$

where the integral spans over the lensing zone whose extension depends on the source intrinsic magnitude and the observational threshold $K_{\text {thr }}$, as mentioned above.

In order to find the total number of sources whose secondary image is above threshold at any given time, we just have to integrate over the source intrinsic magnitude, weighing with the proper luminosity function:

$$
N_{P_{j}}\left(K_{\mathrm{thr}}\right)=\int n_{P_{j}}\left(M_{K}\right) \Pi_{P_{j}}\left(M_{K}, K_{\mathrm{thr}}\right) d M_{K}
$$

Of course we have assumed factorization between the luminosity and spatial distributions. Fig. 1 shows the expected number of sources with detectable secondary image as a function of the threshold magnitude $K_{\mathrm{thr}}$. We can appreciate that the contribution by the cluster P3 is negligible, whereas the bulge roughly yields one order of magnitude more events than the disk and the eccentric cluster P1+P2. At $K_{\mathrm{thr}}=24$ we have 1.4 expected gravitational lensing events; at $K_{\mathrm{thr}}=27$ we have 16 events; at $K_{\text {thr }}=30$ we have 170 events.

In the $V$-band we have similar numbers with $V_{\mathrm{thr}}=K_{\mathrm{thr}}+3$ [13].

\section{Details of the distributions}

A more detailed analysis of the integral (3.2) puts in evidence the relative weight of different contributions to the final result, helping us to understand the phenomenology of gravitational 


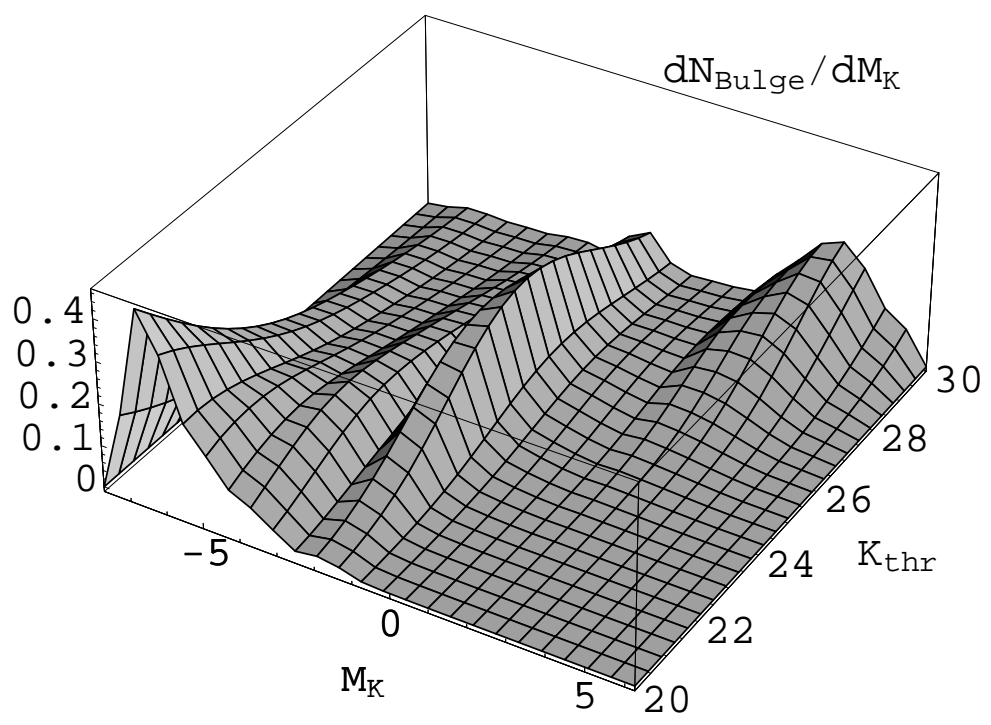

Figure 2: Relative contribution of bulge stars with different absolute magnitudes to the total number of expected events for threshold magnitudes ranging from 20 to 30 in the $K$-band.

lensing by the SMBH and guiding us in the definition of an observational strategy.

Fig. 2 shows the relative contribution of bulge stars with different absolute magnitudes to the total number of events. We can see that observations with small thresholds can only detect secondary images of the brightest stars, lying at the tip of the red giant branch. If the threshold is pushed to higher magnitudes, less luminous stars in the bulge start contributing more and more. We can clearly identify the red clump dominating at $K_{\mathrm{thr}}=24$ and the stars at the turn-off point of the main sequence taking over at $K_{\mathrm{thr}}>28$. A similar analysis can be repeated for all other populations (see [13] for details).

Stars belonging to different populations lie at different distances from the central black hole. The distribution in the source distance shows that stars belonging to P3 are lensed at $0.2 \mathrm{pc}$, stars belonging to $\mathrm{P} 1+\mathrm{P} 2$ are typically lensed at $4 \mathrm{pc}$, stars in the bulge are maximally lensed at $500 \mathrm{pc}$, and stars in the disk at $1.5 \mathrm{kpc}$. Several observables are related to the source distance, e.g. the position of the secondary image.

In Fig. 3 we show the distribution in the angular distance of the secondary images from the central black hole for events generated by sources in the bulge. We can see that secondary images may appear in a wide range of angular radii from the SMBH, from 1 mas to $0.1^{\prime \prime}$. In any case, in order to catch as many images as possible, we need to employ interferometric techniques.

In Fig. 4 we plot the distribution of the angular velocities of the secondary images for sources in the bulge. Most images cover 1 mas every 10 or 100 years. However, there is a long tail extending to smaller and higher velocities. As a consequence, in order to observe any evolution of a gravitational lensing event we need to wait many years. In fact, a secondary image may remain above threshold even some centuries before fading. For the same reason, the rate of new events is also very low [13]. 


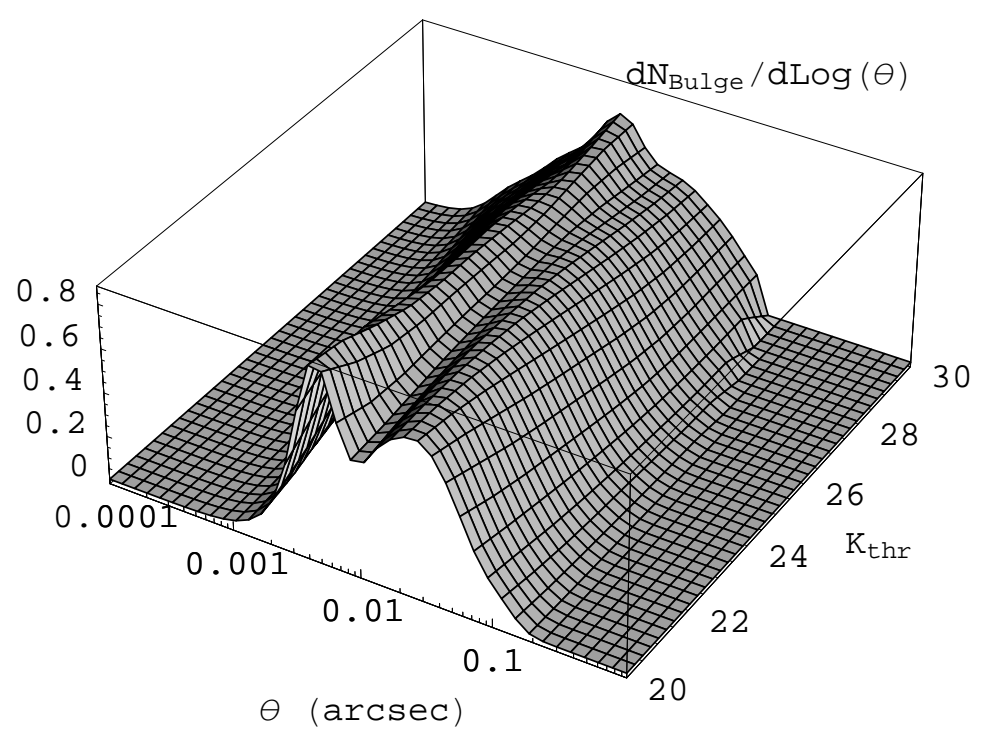

Figure 3: Distributions of the angular distances of the gravitational lensing secondary images from the black hole for stars in the bulge at different values of the threshold magnitude in the $K$-band.

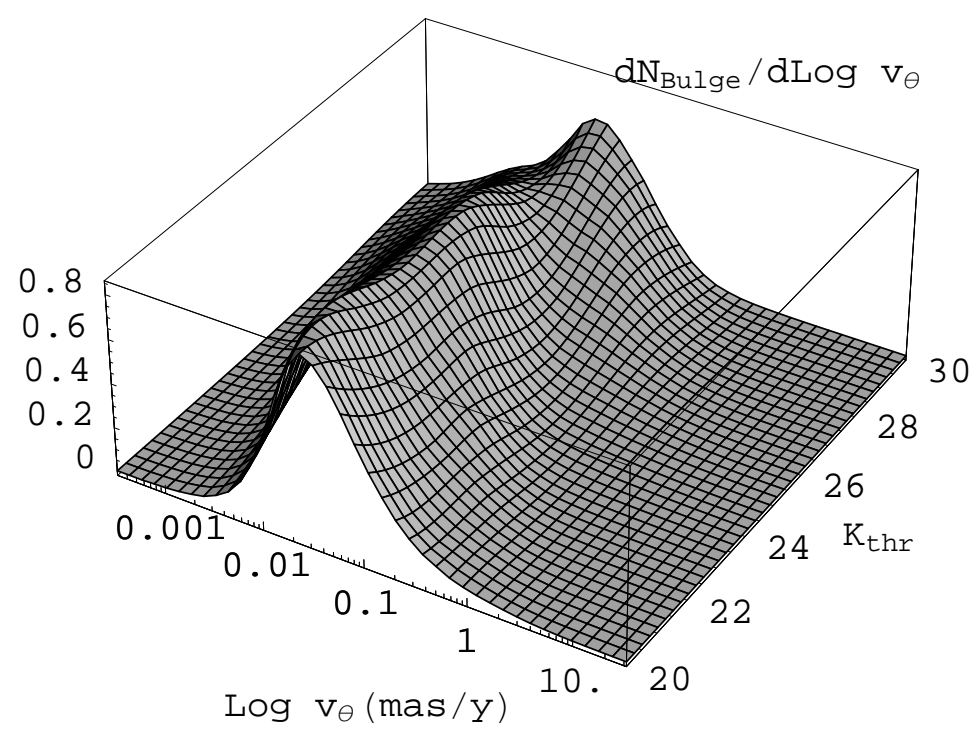

Figure 4: Distributions of the velocities of the gravitational lensing secondary images from the black hole for stars in the bulge at different values of the threshold magnitude in the $K$-band. 


\section{Observational perspectives}

After this thorough discussion of the phenomenology of gravitational lensing by the SMBH in M31, we can draw a possible strategy to distinguish genuine gravitational lensing candidates from background stars.

From our models of the four source populations we can estimate the number of background stars that would show up within a fixed angular radius from the SMBH in an image with limiting magnitude $K_{\mathrm{thr}}$. For example, we find that we have 12 background stars brighter than $K_{\mathrm{thr}}=24$ within $0.01^{\prime \prime}$ from the SMBH. We can select those stars that appears on opposite sides of the black hole as possible candidates for a gravitational lensing event. In order to discard fake alignments, we can take images in different colors and check that the two stars have the same color, since gravitational lensing is an achromatic phenomenon. Finally, a second image can be taken after one year. If the two stars move independently, the alignment should be lost in the second image. If the two stars are really the two images of the same lensed source, the alignment should be preserved in the second image.

Of course, such observations have very high instrumental requirements. We need very high angular resolution, of the order of 1 mas, which can be obtained by large telescopes in interferometric configuration, such as Keck and LBT. Such observations are currently performed in infrared bands, justifying our choice to lead the analysis in the $K$-band. Secondly, we also need very deep imaging in order to increase the chances of observing secondary images. This can be done by the upcoming JWST or by future extremely large telescopes. Therefore, gravitational lensing by the SMBH in M31 has to be considered as a possible project for future advanced telescopes.

Indeed the perspectives opened by the observation of gravitational lensing by SMBHs are extremely interesting. We would get a precise and independent estimate of the mass of the SMBH, which could be used to double-check estimates by dynamical methods. By measurements of the time delay, we can derive the distance to the black hole with very high precision. Observations of gravitational lensing beyond the weak deflection approximation should be possible and could be used to test general relativity in a strong field regime. Finally, if enough statistics of gravitational lensing images is collected, it could be possible to attempt a detailed reconstruction of the mass distribution in the core of the galaxies through gravitational lensing. In conclusion, we can say that gravitational lensing by SMBH would provide a considerable amount of independent information on the galactic cores. In this work we have shown that such an opportunity will be within range of realistic future telescopes.

\section{References}

[1] N. Häring and H.-W. Rix, On the Black Hole Mass-Bulge Mass Relation, ApJ 604 (2004) L89 [astro-ph/0402376].

[2] F. Eisenhauer et al., SINFONI in the Galactic Center: Young Stars and Infrared Flares in the Central Light-Month, ApJ 628 (2005) 246 [astro-ph / 0502129$].$

[3] M. Wardle and F. Yusuf-Zadeh Gravitational lensing by a massive black hole at the Galactic center, ApJ 387 (1992) L65. 
[4] M. Jaroszyński, Gravitational Lensing and Proper Motions of Stars Surrounding the Galactic Center, Acta Astron. 48 (1998) 413.

[5] T. Alexander and A. Sternberg, Near-Infrared Microlensing of Stars by the Supermassive Black Hole in the Galactic Center, ApJ 520 (1999) 137 [astro-ph/9811038].

[6] J. Chanamé, A. Gould and J. Miralda-Escudé, Microlensing by Stellar Black Holes around Sagittarius $A^{*}$, ApJ 563 (2001) 793 [astro-ph/ 0102481$]$.

[7] T. Alexander, Pinpointing the Massive Black Hole in the Galactic Center with Gravitationally Lensed Stars, ApJ 553 (2001) L149 [astro-ph / 0102055$].$

[8] A. Nusser and T. Broadhurst, Monitoring lensed starlight emitted close to the Galactic centre, MNRAS 355 (2004) L6 [astro-ph/ 0407220$].$

[9] F. De Paolis, A. Geralico, G. Ingrosso and A.A. Nucita, The black hole at the galactic center as a possible retro-lens for the S2 orbiting star, A\&A 409 (2003) 809 [astro-ph/ 0307493 ].

[10] V. Bozza and L. Mancini, Gravitational Lensing by Black Holes: A Comprehensive Treatment and the Case of the Star S2, ApJ 611 (2004) 1045 [astro-ph / 0404526 ].

[11] V. Bozza and L. Mancini, Gravitational Lensing of Stars in the Central Arcsecond of Our Galaxy, ApJ 627 (2005) 790 [astro-ph/0503664].

[12] C.R. Keeton and A.O. Petters, Formalism for testing theories of gravity using lensing by compact objects: Static, spherically symmetric case, Phys. Rev. D 72 (2005) 104006 [gr-qc/ 0511019 ].

[13] V. Bozza, S. Calchi Novati and L. Mancini, Gravitational lensing by the supermassive black hole in M31, ApJ 675 (2008) 340 [arXiv: 0711 . 0750].

[14] T.R. Lauer et al., Planetary camera observations of the double nucleus of M31, AJ 106 (1993) 1436.

[15] S. Tremaine, An Eccentric-Disk Model for the Nucleus of M31, AJ 110 (1995) 628 [astro-ph/9502065].

[16] R. Bender et al., HST STIS Spectroscopy of the Triple Nucleus of M31: Two Nested Disks in Keplerian Rotation around a Supermassive Black Hole, ApJ 631 (2005) 280 [astro-ph/ 0509839 ].

[17] A. Aparicio and C. Gallart, IAC-STAR: A Code for Synthetic Color-Magnitude Diagram Computation, AJ 128 (2004) 1465 [astro-ph/ 0407589 ].

[18] H.V. Peiris and S. Tremaine, Eccentric-Disk Models for the Nucleus of M31, ApJ 599 (2003) 237 [astro-ph/0307412].

[19] S.M. Kent, An improved bulge model for M31, AJ 97 (1989) 1614.

[20] A. Sarajedini and P. Jablonka, The Metallicity Distribution Function of Field Stars in M31's Bulge, AJ 130 (2005) 1627 [astro-ph / 0506653 ].

[21] L.M. Widrow and J. Dubinski, Equilibrium Disk-Bulge-Halo Models for the Milky Way and Andromeda Galaxies, ApJ 631 (2005) 838 [astro-ph/ 0506177 ].

[22] M. Bellazzini et al., The Andromeda project. I. Deep HST-WFPC2 V, I photometry of 16 fields toward the disk and the halo of the M 31 galaxy. Probing the stellar content and metallicity distribution, A\&A 405 (2003) 867 [astro-ph/0212531]. 